\section{J'TI}

JOURNAL OF

TRAUMA AND INJURY

Received: July 5, 2021

Revised: August 5, 2021

Accepted: August 10, 2021

\section{Correspondence to}

Chan Yong Park, M.D., Ph.D.

Division of Trauma Surgery, Department of Surgery, Seoul National University

Hospital, 101 Daehak-ro, Jongno-gu,

Seoul 03080, Korea

Tel: + 82-2-2072-2817

Fax: +82-2-766-3975

E-mail: trauma-park@naver.com

ORCID: https://orcid.org/0000-0002-

5111-3270

\title{
Pre-Hospital and In-Hospital Manage- ment of an Abdominal Impalement Injury Caused by a Tree Branch
}

\author{
So Ra Ahn, M.D. ${ }^{1}$, Joo Hyun Lee, M.D. ${ }^{2}$, Keun Young Kim, M.D. ${ }^{2}$, \\ Chan Yong Park, M.D., Ph.D. ${ }^{3}$ \\ ${ }^{1}$ Department of Trauma Surgery, Wonkwang University Hospital Regional Trauma Center, \\ Iksan, Korea \\ ${ }^{2}$ Department of Surgery, Wonkwang University School of Medicine, Iksan, Korea \\ ${ }^{3}$ Division of Trauma Surgery, Department of Surgery, Seoul National University Hospital, \\ Seoul, Korea
}

In South Korea, most patients who visit trauma centers with abdominal injuries have blunt trauma, and penetrating injuries are relatively rare. In extremely rare cases, some patients are admitted with a long object penetrating their abdomen, and these injuries are referred to as abdominal impalement injuries. Most cases of impalement injuries lead to fatal bleeding, and patients often die at the scene of the accident. However, patients who survive until reaching the hospital can have a good prognosis with optimal treatment. A 68-year-old female patient was admitted to the trauma center with a 4-cm-thick tree branch impaling her abdomen. The patient was transported by a medical helicopter and had stable vital signs at admission. The branch sticking out of the abdomen was quite long; thus, we carefully cut the branch with an electric saw to perform computed tomography (CT). CT revealed no signs of major blood vessel injury, but intestinal perforation was observed. During laparotomy, the tree branch was removed after confirming that there were no vascular injuries, and enterostomy was performed because of extensive intestinal injury. After treating other injuries, the patient was discharged without any complications except colostomy. Abdominal impalement injuries are treated using various approaches depending on the injury mechanism and injured region. However, the most important consideration is that the impaled object should not be removed during transportation and resuscitation. Instead, it should only be removed after checking for injuries to blood vessels during laparotomy in an environment where injury control is possible.

Keywords: Abdominal injuries; Penetrating; Impalement; Laparotomy 


\section{INTRODUCTION}

Abdominal injuries are categorized as blunt or penetrating injuries. In South Korea, which prohibits personal possession of guns, most abdominal injuries are blunt injuries caused by traffic accidents and falls, but stab wounds from sharp objects such as knives and glass fragments also occur occasionally. Penetrating injuries in which a long object impales the trunk are referred to as impalement injuries [1,2]. Although impalement injuries are extremely rare, they may be fatal because of their potential damage to the abdominal organs and vasculature [3-5]. Herein, we report a case of a typical abdominal impalement injury and review the pre-hospital care, transport, and treatment in the trauma bay and operating room.

\section{CASE REPORT}

A 68-year-old female was admitted with a tree branch impaling her abdomen. The patient was in the passenger seat of a 1-ton truck that rear-ended a stopped vehicle carrying a large tree. When the paramedics arrived at the scene, a tree branch was found to have impaled her abdomen (Fig. 1). The patient was transported by a medical helicopter and arrived at the hybrid emergency room of the regional trauma center of Wonkwang University Hospital 70 min-

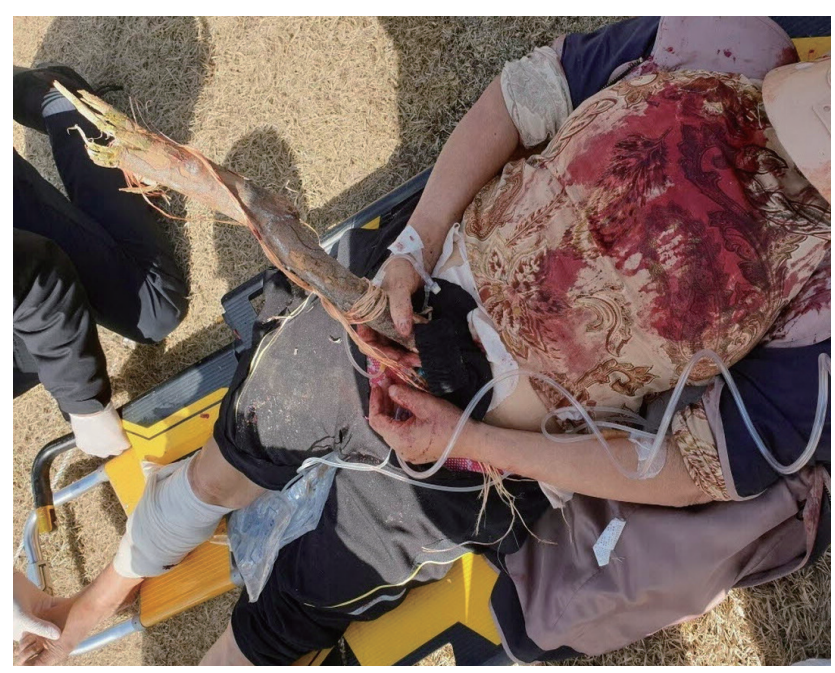

Fig. 1. 119 rescue on-site: the patient with a tree branch impaling the abdomen is being prepared for transport by a helicopter. utes after the accident. A 4-cm-thick tree branch was impaled at a $45^{\circ}$ angle into the left side of the umbilicus, and approximately $50 \mathrm{~cm}$ of the branch protruded outside (Fig. 2). There was no protrusion of the bowel or mesentery around the penetrating site, and the patient had no active bleeding. In addition, a firm foreign body was palpated inside the left flank, but it did not protrude (i.e., there was no exit site). There were no signs of peritonitis, such as tenderness or rebound tenderness in the abdomen. The patient's Glasgow Coma Scale score was 14 at the time of admission, and her vital signs were as follows: blood pressure (BP) 110/60 mmHg; pulse rate, 93 beats/ minute. In response to a potential main vessel injury, a blood sample was taken immediately upon admission, and emergency blood transfusion was administered with two units of universal $\mathrm{O}^{+}$packed red blood cells. The initial hemoglobin level was $9.1 \mathrm{~g} / \mathrm{dL}$. The part of the tree branch protruding outside the patient's body was quite long and hindered the performance of computed tomography (CT). Hence, we carefully cut the tree branch with an electric saw for CT (Fig. 3), and the patient's BP remained stable. The patient also had swelling around the right eye, multiple lacerations on the face, and a $20 \times 10$ $\mathrm{cm}$ degloving injury on the right leg. Her vital signs were stable, and CT could be performed immediately. Abdominal CT revealed a penetrating injury caused by a foreign body in the left lower quadrant, and the foreign body

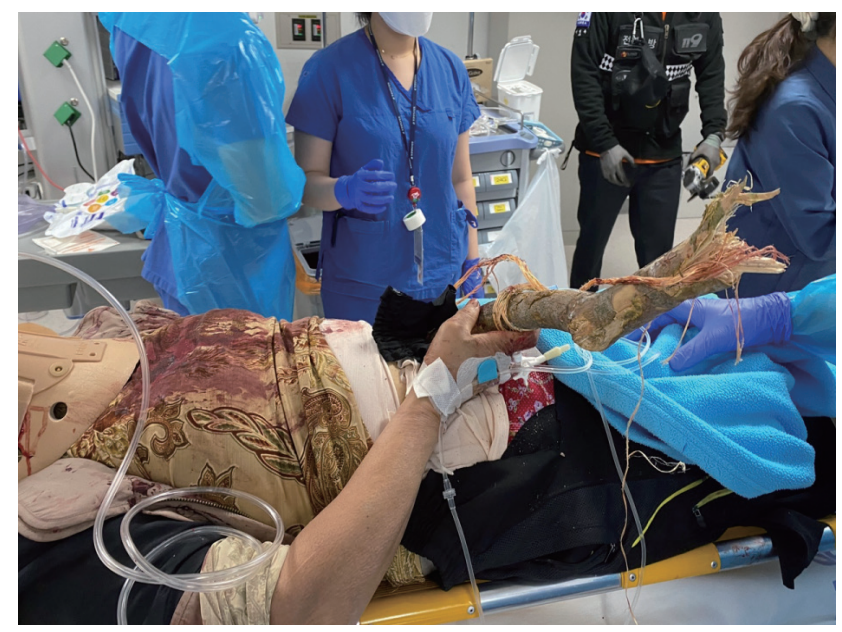

Fig. 2. The patient was admitted with a tree branch impaling the lower abdomen; no intraabdominal organs protruded outside the body, and there was no active bleeding. 


\section{JTI}

perforated the bowel and was positioned between the left 11 th and 12th ribs. Fortunately, there were no signs of major vessel injuries in the abdominal cavity (Fig. 4). Facial CT revealed fractures in the midfacial bone and right sphenoid bone. Vascular CT of the right lower extremity revealed a soft tissue defect in the calf, without any vascular injury. Due to peritoneal contamination with a tree branch, empirical broad-spectrum antibiotics and tetanus vaccine were administered preoperatively. After catheterization with a C-line and A-line, the patient was transferred to the operating room 1 hour after arrival. Under general anesthesia and with the tree branch not removed, a midline laparotomy was performed. There was no significant intraperitoneal bleeding, and penetration of the descending colon and sigmoid colon and mesenteric laceration of the transverse colon were observed (Fig. 5). The distal end of the tree branch was impaled under the left 11 th rib, and no splenic injury was noted. After confirming that there were no injuries to the aorta or mesenteric vessels, the foreign body was removed from the outside (Fig. 6). Although fecal contamination in the abdominal cavity was not severe, there was an extensive injury to the colon and the colon was filled with large amounts of hard stool. Therefore, to prevent anastomosis leakage,
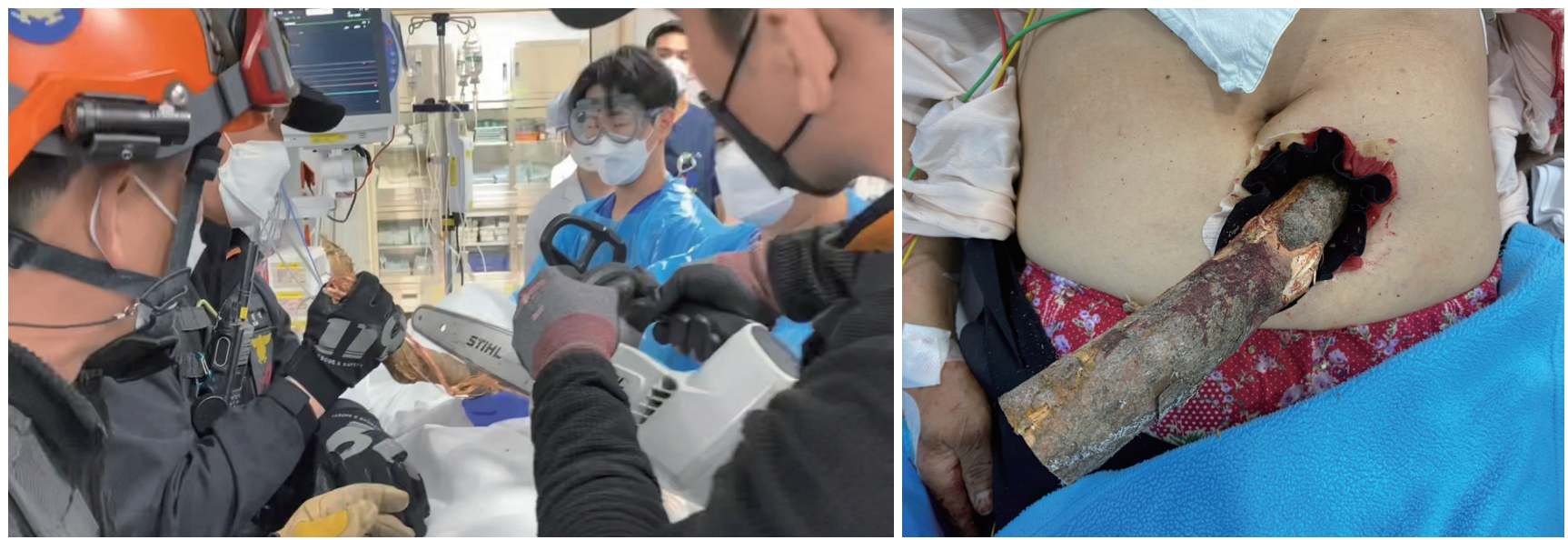

Fig. 3. The protruding portion of a tree branch was excessively long to perform computed tomography (CT); thus, we carefully cut it with an electric saw before CT.
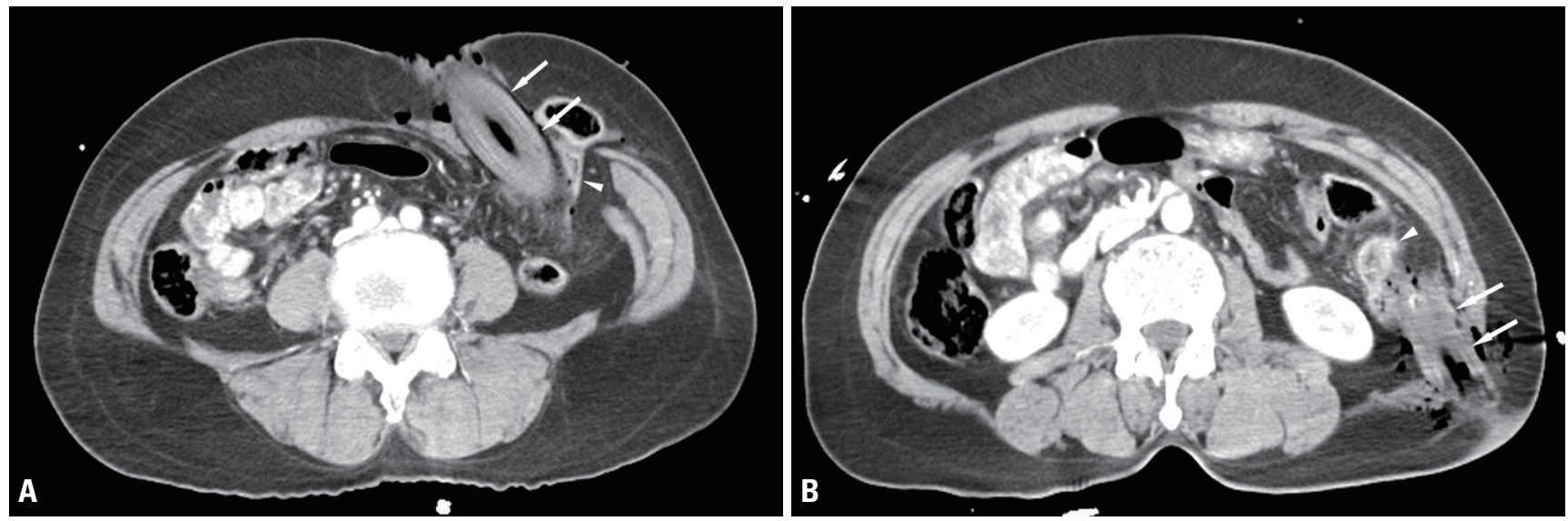

Fig. 4. (A, B) Abdomen computed tomography revealed a penetration injury in the left lower quadrant caused by a foreign body (white arrows). The foreign body perforated the bowel (arrowhead) and was placed between the left 11th and 12th ribs. Fortunately, there were no major vessel injuries in the abdominal cavity. 

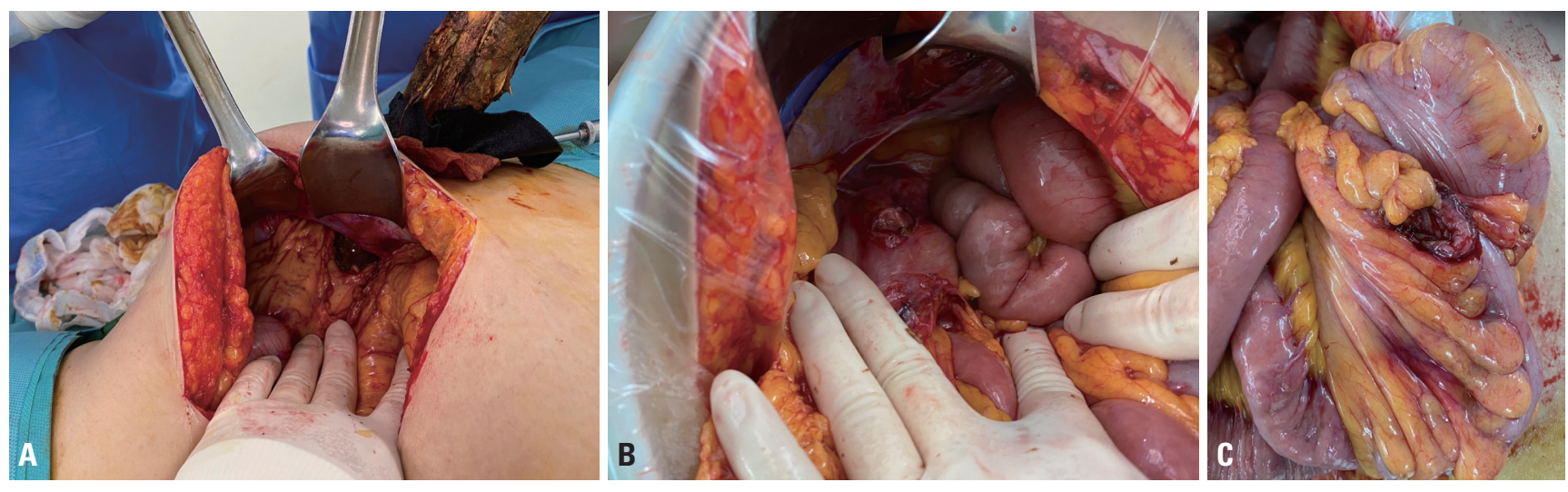

Fig. 5. (A) A midline incision was made, and there was little bleeding from the intraabdominal cavity. $(B, C)$ Penetration of the descending colon and sigmoid colon and laceration of the T-colon mesentery were observed.

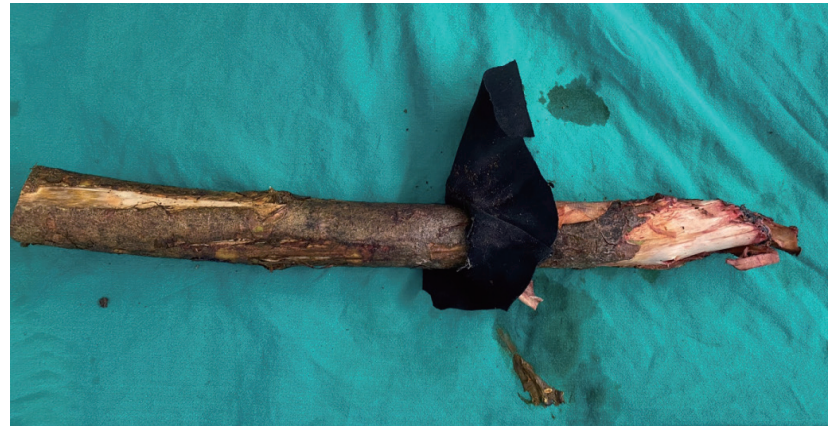

Fig. 6. Tree branch removed from the abdomen.

an end-colostomy of the transverse colon was performed after left hemicolectomy. After meticulous irrigation, the abdominal wall tract impaled by the foreign body and the dorsal peritoneal injury were repaired. Additionally, the patient underwent irrigation, drainage, and augmentation for the degloving injury of the right leg during orthopedic surgery and was admitted to the intensive care unit. On day 8 of hospital admission, the patient underwent surgery to treat the facial bone fracture through oral and maxillofacial surgery. Several rounds of negative-pressure wound therapy were performed, and the skin defect was treated with a skin graft. The patient was discharged on day 58 of hospital admission and was scheduled for colostomy repair.

This study was approved by the Institutional Review Board (IRB) of the Wonkwang University Hospital (IRB No. 2021-06-018).

\section{DISCUSSION}

Abdominal injuries observed at trauma centers in Korea are predominantly caused by blunt trauma due to traffic accidents and falls. However, some patients sustain penetrating injuries from a knife or sharp object and are admitted with intraperitoneal bleeding. A penetrating injury in which the object remains impaled in the body is referred to as an impalement injury. Although most patients who sustain a penetrating injury are admitted after the penetrating object is removed, some patients are admitted with a short cutting blade or other weapons stuck in their bodies $[3,4]$. However, patients admitted with a thick object impaled in the abdomen, as this case, are rare. Impalement injuries, which occur mostly due to traffic accidents, falls, homicide, and accidents at construction sites, are classified as a type of penetrating injury, but their mechanism involves both blunt and penetrating injuries. Thus, extensive tissue, vascular, and deep organ injuries and foreign body contamination can occur [3-7]. In particular, an impalement injury of the torso, such as the abdomen, can be fatal, as it can damage major vessels, such as the aorta and inferior vena cava, and solid organs and hollow viscus organs. If major vessels in the abdominal cavity are injured, the patient will most likely die at the scene of the accident. If a patient is able to survive until reaching the hospital, the patient has a good chance of survival with optimal treatment $[3,8]$.

Because impalement injuries are rare and vary widely 
in terms of the area and form of injury depending on the mechanism, there is no standard protocol for their management. However, certain rules exist. The most important rule for an abdominal impalement injury is that the impaled object must not be removed at the scene or during transport. This is because the object may be compressing injured vessels and stopping them from bleeding. Therefore, the object should be removed after checking for injuries to blood vessels during laparotomy in an environment where injury control is possible $[3,8,9]$. However, if the object is quite long, to the point that it hinders the transport of the patient, it can be cut short. When cutting the object is unavoidable, it is important to be very careful not to cause a secondary injury to intraabdominal organs due to shaking or vibrations of the object. In cases of metal impalement injury, a hydraulic pedal cutter is usually used to minimize secondary damage caused by vibration $[1,8]$. However, wood (as in our case) is difficult to cut with a pedal cutter, so it can be cut quickly and safely with a chainsaw after fixing the wood firmly. Furthermore, selecting the quickest and most stable method of transport is crucial for a good prognosis. During transport, the impaled object must be fixed such that it does not move, and the patient's initial posture at the time of injury should be maintained to the extent possible. If possible, as in this case, transport by helicopter can help to prevent vibrations and shorten the transport time $[1,8,10,11]$.

When a patient with an abdominal impalement injury arrives at the hospital, the treatment depends on the patient's vital status. Hemodynamically unstable patients should be transferred to the operating room immediately without imaging tests to perform laparotomy and check the intraperitoneal state carefully while ensuring hemostasis. In contrast, contrast-enhanced CT is the gold-standard diagnostic modality if the patient is hemodynamically stable during and after transport, as in this case. CT can reveal injuries of the blood vessels, abdominal organs, and other regions, and it is important to understand the trajectory of the object to anticipate and prepare for potential damage before surgery $[3,4,12,13]$. Based on these findings, surgeons can collaboratively develop a surgical plan. If the impaled object is quite long and hinders the performance of CT, cutting the object using a method with minimal vibration and movement is helpful. Most impalement injuries involve multi-organ injuries; therefore, a multidisciplinary team is essential. The impaled object must be removed only after confirming intraperitoneal injuries and appropriately controlling for vascular injuries. In addition, the object penetrating the abdomen is contaminated; therefore, preoperative administration of broad-spectrum empirical antibiotics and tetanus vaccine is necessary $[4,6,8,11]$.

There are some caveats to keep in mind when treating penetrating wounds caused by trees. Wood foreign objects are contaminating and contagious because the porous organic nature of wood provides excellent culture conditions for Gram-positive bacteria, such as Staphylococcus epidermis and Staphylococcus aureus, as well as Gram-negative bacteria. Infections with these micro-organisms can cause severe sepsis, cellulitis, and abscess formation. Even clean wood can cause a foreign body reaction. Therefore, it is very important to remove foreign objects early and to clean the area carefully to avoid leaving debris such as projectile particles, dust, and micro-organisms such as bacteria. An empirical broad-spectrum antibiotic must also be administered [14-16].

\section{CONCLUSION}

Abdominal impalement injuries are rare, but can be fatal. In most cases, the patient dies at the scene, but if the patient survives until reaching the hospital, optimal treatment can save the patient's life. The treatment depends on the mechanism and region of injury, but the most important consideration is not to remove the impaled object and to transport the patient to the hospital with minimal movement. The impaled object should be removed after checking for injuries to blood vessels during laparotomy in an environment where injury control is possible.

\section{CONFLICTS OF INTEREST}

No potential conflict of interest relevant to this article was reported. 


\section{INFORMED CONSENT}

Informed consent was obtained from all individual participants included in this study.

\section{REFERENCES}

1. Lee SY, Lee JM, Choi SJ. Abdominal impalement injury caused by scaffolding pipe following a traffic accident: a case report. J Trauma Inj 2016;29:33-6.

2. Noh HN, Kim KM, Park JB, Ryu H, Bae KS, Kang SJ. Clinical analysis of patients with abdomen or neck-penetrating trauma. J Korean Soc Traumatol 2010;23:107-12.

3. Ugoletti L, Zizzo M, Castro Ruiz C, Pavesi E, Biolchini F, Annessi V. Gluteal, abdominal, and thoracic multiple impalement injuries: a case report on management of a complex polytrauma. Medicine (Baltimore) 2019;98:e15824.

4. Mohan R, Ram DU, Baba YS, Shetty A, Bhandary S. Transabdominal impalement: absence of visceral or vascular injury a rare possibility. J Emerg Med 2011;41:495-8.

5. Byard RW. A forensic evaluation of impalement injuries. Med Sci Law 2018;58:85-92.

6. Djelti A, Jneid H. Survival after abdominal impalement with a diver's harpoon. Int J Surg Case Rep 2020;68:270-2.

7. Bergaminelli C, Salvi R, Mattiacci DM, Messina G, Cicalese M,
Curcio C, et al. Management of chest impalement injury. Int J Surg Case Rep 2019;61:123-6.

8. Jawaid S, Cody D. Crowbar impalement: the PHEM perspective. BMJ Case Rep 2018;11:e227293.

9. Yang XW, Wang WT. Chest, pericardium, abdomen, and thigh penetrating injury by a steel rebar: a case report. World J Clin Cases 2020;8:5025-9.

10. Sawhney C, D'souza N, Mishra B, Gupta B, Das S. Management of a massive thoracoabdominal impalement: a case report. Scand J Trauma Resusc Emerg Med 2009;17:50.

11. Thomson BN, Knight SR. Bilateral thoracoabdominal impalement: avoiding pitfalls in the management of impalement injuries. J Trauma 2000;49:1135-7.

12. Biffl WL, Leppaniemi A. Management guidelines for penetrating abdominal trauma. World J Surg 2015;39:1373-80.

13. Vaslef SN, Dragelin JB, Takla MW, Saliba EJ Jr. Multiple impalement with survival. Am J Emerg Med 1997;15:70-2.

14. Singh RK, Bhandary S, Karki P. Managing a wooden foreign body in the neck. J Emerg Trauma Shock 2009;2:191-5.

15. Dalley RW. Intraorbital wood foreign bodies on CT: use of wide bone window settings to distinguish wood from air. AJR Am J Roentgenol 1995;164:434-5.

16. Vakil MT, Singh AK. A review of penetrating brain trauma: epidemiology, pathophysiology, imaging assessment, complications, and treatment. Emerg Radiol 2017;24:301-9. 